\title{
Fremdsprachen performativ lehren und lernen
}

\author{
Manfred Schewe \\ Department of German, University College Cork, Ireland \\ m.schewe@ucc.ie
}

\begin{abstract}
Zusammenfassung
Dieser Beitrag ist als Einführung in das performative Lehren und Lernen zu verstehen. Zunächst wird der Unterschied zwischen einem performativ und kommunikativ gestalteten Fremdsprachenunterricht kurz skizziert. Dazu gehört insbesondere, dass im performativen Konzept eine enge Anbindung an die Künste zentral ist und der Körper als wichtiges Kommunikations- und Erkenntnismedium angesehen wird. Die Darstellung von charakteristischen Merkmalen einer performativen Lehr- und Lernkultur mündet in den Umriss einer performativen Fremdsprachendidaktik, in der die Drama- und Theaterpädagogik als zwischen Wissenschaft und Kunst vermittelnde Disziplinen zu Kerndisziplinen avancieren. Anhand einer Grafik werden die Grundannahmen einer solchen Didaktik veranschaulicht und erläutert. Es folgt ein Überblick über das inzwischen breite Spektrum von performativen Ansätzen im sprach-, literatur- und kulturbezogenen Fremdsprachenunterricht, wobei zwischen performativen Groß- und Kleinformen unterschieden wird. Der Beitrag endet mit Hinweisen auf aktuelle Forschungsarbeiten und auf Bereiche, in denen künftig weiter geforscht werden sollte.

Schlüsselwörter: performativ, Kunst, künstlerisch, ästhetisch, Dramapädagogik, performative Fremdsprachendidaktik
\end{abstract}

\begin{abstract}
This contribution serves as a short introduction to performative teaching and learning. It begins with a brief outline of the difference between communicative and performative foreign language and learning. This includes a strong emphasis, within the performative concept, on a close connection with the arts and especially on forms of embodied teaching and learning. Some typical features of a performative teaching and learning culture are presented, followed by an outline of a performative foreign language didactics; within this theoretical framework drama and theatre pedagogy, as bridge builders between the Sciences and the Arts, function as core disciplines. Reference is
\end{abstract}


SCHEWE, Manfred - Fremdsprachen performativ lehren und lernen. Para lá da tarefa: implicar os estudantes na aprendizagem de línguas estrangeiras no ensino superior. Porto: FLUP, 2019, pp. 343-361 DOI: https://doi.org/10.21747/9789898969217/paraa19

then made to a graphic representation to illustrate and explain in more detail the basic ideas of a performative didactics; furthermore, an overview is given over the broad range of performative approaches to language-, literature- and culture-based foreign language teaching and learning that have emerged in recent years. In doing so a distinction is made between large and small scale formats. Finally, reference is made to current research projects and to research areas which should be explored further in the years to come.

Keywords: performative, the Arts, artistic, aesthetic, drama pedagogy, performative foreign language didactics.

\section{1 - Einleitung: Von ,kommunikativ‘ zu ,performativ‘}

Seit einigen Jahrzehnten ist in den modernen Fremdsprachen der Begriff ,kommunikativ" zentral. Viele Lehrerinnen und Lehrer weltweit empfanden den kommunikativen Fremdsprachenunterricht (Schumann, 2010) als große Befreiung, denn der in vielen Institutionen noch von der Grammatik-Übersetzungsmethode bzw. audiolingualen Methode stark geprägte Fremdsprachenunterricht versprach von nun an lebendiger zu werden. Der lehrperson- und sprachstrukturzentrierte traditionelle Unterricht wurde kritisch hinterfragt und es reifte die Erkenntnis, dass man eine Sprache in erster Linie lernt, um Inhalte verstehen und ausdrücken zu können. Situation, kommunikatives Bedürfnis oder auch Text wurden zunehmend zu Ausgangspunkten für die Unterrichtsgestaltung, absolute sprachliche Korrektheit war nicht mehr das oberste Ziel, vielmehr, dass die Lernenden Redeabsichten kommunikativ umsetzen können. Wenn auch unter dem Begriff ,kommunikativ“ nicht immer das Gleiche verstanden wird und die Diskussion über die Ursprünge der kommunikativen Wende in den modernen Fremdsprachen noch nicht abgeschlossen ist (Schmenk, 2017), ist es doch ein bleibendes Verdienst der kommunikativen Didaktik, dass ab den 70er/80er Jahren der alltägliche Fremdsprachenunterricht lernerzentrierter und interaktiver wurde. Huber (2003, p. 1) gibt allerdings zu bedenken:

Den kommunikativen Methoden zum Trotz entwickelt sich nur eine Minderheit schulischer Fremdsprachenlerner $\mathrm{zu}$ engagiert in eigener Sache Kommunizierenden, zu den kompetenten und konversationell flexiblen Sprechern, die den Gründervätern der kommunikativen Didaktik beim großen reformpädagogischen Aufbruch Anfang der 70er Jahre vorgeschwebt haben 
SCHEWE, Manfred - Fremdsprachen performativ lehren und lernen. Para lá da tarefa: implicar os estudantes na aprendizagem de línguas estrangeiras no ensino superior. Porto: FLUP, 2019, pp. 343-361 DOI: https://doi.org/10.21747/9789898969217/paraa19

mochten. Das damals formulierte Ideallernziel der kommunikativen Kompetenzdies das ernüchternde Fazit von drei Jahrzehnten kommunikativer Unterrichtspraxis - ist ein unerreichbares Ideal geblieben.

Huber vermisst in der kommunikativen Didaktik vor allem eine künstlerische Dimension. In ihrem an der Universität Lissabon durchgeführten Forschungsprojekt Im Haus der Sprache wohnen. Wahrnehmung und Theater im Fremdsprachenunterricht stellt sie praxisbezogene Modelle eines „aisthetisch-ästhetisch orientierten Fremdsprachenunterrichts" (2003, p. 3) vor und gehört damit zu den Fremdsprachendidaktikerinnen und -didaktikern, die im Bereich Deutsch als Fremdsprache erste Weichen für die Entwicklung einer performativen Fremdsprachendidaktik gestellt haben.

In diesem fachhistorischen Zusammenhang sind weitere Forschungsarbeiten zu nennen, welche die Aufmerksamkeit auf die Verbindung zwischen darstellender Kunst und Pädagogik richteten und die Dramapädagogik (Drama in Education) als neue Bezugsdisziplin für die Fremdsprachendidaktik ins Spiel brachten. Beispielhaft seien hier einige Titel genannt: Fremdsprache inszenieren. Zur Fundierung einer dramapädagogischen Lehr- und Lernpraxis (Schewe, 1993), Dramapädagogik im Sprachunterricht (Tselikas, 1998) und Drama Grammatik. Dramapädagogische Ansätze für den Grammatikunterricht Deutsch als Fremdsprache (Even, 2002).

Die Dramapädagogik lenkte den Blick auf das ,Drama als eine pädagogische Kunstform' und damit auf eine Dimension von Unterricht, die in der kommunikativen Didaktik unterbelichtet geblieben war, nämlich auf das enorme Potenzial körperbezogenen Lernens und Lehrens. Sie wird u.a. mit der Aktivierung möglichst vieler Sinne und der Nutzung aller zur Verfügung stehenden Ausdrucksmittel in fiktiven Handlungskontexten assoziiert. Ihre ganzheitliche Orientierung spiegelt sich in dem Leitsatz: „Im dramapädagogischen Fremdsprachenunterricht wird mit Kopf, Herz, Hand und Fuß gelernt und gelehrt" (Schewe, 1993, p. 8; s. darin besonders auch das Kapitel ,Unterricht als sinnliche Gestaltung'). 
SCHEWE, Manfred - Fremdsprachen performativ lehren und lernen.

Para lá da tarefa: implicar os estudantes na aprendizagem de línguas estrangeiras no ensino superior.

Porto: FLUP, 2019, pp. 343-361

DOI: https://doi.org/10.21747/9789898969217/paraa19

\section{2 - Moderne Fremdsprachen im Spannungsfeld von Wissenschaft und Kunst}

Ein Blick in die Satzungen von Berufsverbänden im Bereich der Modernen Fremdsprachen genügt, um festzustellen, dass das Lehren und Lernen fast ausschließlich aus wissenschaftlicher Perspektive betrachtet wird. Zwar wird schon länger die Position vertreten, dass das Lehren und Lernen von Fremdsprachen nicht nur eine Wissenschaft, sondern ebenso eine Kunst ist (z.B. Schewe, 1997), aber erst seit einigen Jahren scheint das Interesse an einer stärkeren Auseinandersetzung mit dieser Position zuzunehmen. Ein Grund dafür mag sein, dass sich derzeit Wissenschaft und Kunst auch in Kontexten außerhalb der Fremdsprachen stärker aufeinander zu bewegen. So legt es etwa der Artikel 'Welche Sprachen spricht die Kunst?' von Sybille Anderls (2019) nahe, der mit dem Satz beginnt: «Das alte Liebespaar Wissenschaft und Kunst hat wieder zueinander gefunden». Darin bezieht sie sich u.a. auf den Informatiker Ben Shneiderman, der im Rahmen eines interdisziplinären Kolloquiums an der National Academy of Sciences in Washington die Ansicht vertrat:

Wissenschaftler könnten von Künstlern eine feinsinnigere Wahrnehmung lernen. Die Visionen von Künstlern würden darüber hinaus neue wissenschaftliche und technologische Entwicklungen anregen. Die breit gefächerte, explorative und verspielte methodische Herangehensweise von Künstlern sei außerdem geeignet, das Denken der Wissenschaftler zu weiten. Und schließlich könne man auch auf eine direkte Inspiration der Forschenden durch Kunstwerke hoffen. Wenn all diese Mutmaßungen stimmen, kann die Wissenschaft ihre Erkenntnisfähigkeit unter künstlerischer Einflussnahme nur verbessern. (Anderl, 2019)

Solcherart Überlegungen, angewendet auf den Bereich Moderne Fremdsprachen, führen mich zu der hypothetischen Überlegung: Da die Fremdsprachendidaktik «ihre Erkenntnisfähigkeit unter künstlerischer Einflussnahme nur verbessern» kann, ist es konsequent, dass sie sich zu den Künsten hin weiter öffnet und anstrebt, sich langfristig als eine wissenschaftlich-künstlerische Disziplin zu etablieren. Zu diesem Zwecke müsste sie damit beginnen, die produktive Wechselbeziehung von Wissenschaft und Kunst systematischer zu untersuchen. Es liegen bereits Publikationen vor, die den Weg dorthin ebnen helfen. Beispielhaft seien hier einige genannt: Bernstein/Lerchner (2014) beleuchten, wie durch den Einsatz von Literatur, Theater, Bildender Kunst, Musik und Film im Fremdsprachenunterricht ästhetische Erkenntnis ermöglicht; Moraitis et al. 
SCHEWE, Manfred - Fremdsprachen performativ lehren und lernen. Para lá da tarefa: implicar os estudantes na aprendizagem de línguas estrangeiras no ensino superior. Porto: FLUP, 2019, pp. 343-361 DOI: https://doi.org/10.21747/9789898969217/paraa19

(2018) stellen in ihrem Sammelband verschiedene Konzepte und Methoden von ästhetischem Lernen und (Fremd-) Sprachenerwerb bzw. Erziehung und Bildung vor; Mentz \& Fleiner (2018) nähern sich dem Thema 'The Arts in Language Teaching' aus internationaler Perspektive; Piazzoli (2018) und Woodhouse \& Schewe (2018) liefern Überlegungen zum Lehren als einer künstlerischen Tätigkeit und Hensel (in Vorbereitung) setzt sich in ihrem Dissertationsprojekt an der FU Berlin mit dem Thema ,Fremdsprachenunterricht als Ereignis - Zur Fundierung einer performativ-ästhetischen Praxis' auseinander.

Weitere Impulse werden voraussichtlich von dem Kongress 2019 der Deutschen Gesellschaft für Fremdsprachenforschung (DGFF) mit dem Fokus auf 'Sprachen, Kulturen, Identitäten: Umbrüche durch Digitalisierung?' ausgehen, denn auf dem Kongress wird unter 'Freie Formate' auch das Thema Die Künste und der Fremdsprachenunterricht - Schnittflächen \& Perspektiven vertreten sein (s. Link in Literaturliste). Unsere Bildungssysteme, einschließlich der Fremdsprachenpädagogik, müssen auf gesellschafts- und weltpolitische Entwicklungen reagieren. Die Menschheit im 21. Jahrhundert steht vor enormen Herausforderungen (vgl. Link in Literaturliste zu Sustainable Development Goals der UN). Kreative Lösungen sind gefragt, aber Kreativität wird bislang an Hochschulen und Schulen nicht ausreichend gefördert und eine künstlerisch orientierte Neuausrichtung in Lehre und Forschung scheint daher dringend erforderlich. Ganz im Sinne der UNESCO-Weltkongresse Lissabon 2006 und Seoul 2010 (s. Links in Literaturliste) muss es fortan darum gehen, die Rolle der Künste in der Bildung zu stärken. Dieses Ziel verfolgt die bilinguale (Deutsch-Englisch) Fachzeitschrift Scenario (http://scenario.ucc.ie), indem sie sich seit ihrer Gründung im Jahr 2007 für einen Brückenbau zwischen den performativen Künsten und Pädagogik engagiert und die Entwicklung einer performativen Lehr- und Lernkultur vorantreibt.

\section{3 - Zur Charakteristik einer performativen Lehr- und Lernkultur}

Was unter einer performativen Lehr- und Lernkultur zu verstehen ist, wurde auf dem 6. Scenario Forum Symposium (Universität Hannover, 21.-22.9. 2018) erarbeitet (Jogschies, Schewe \& Stöver-Blahak, 2018). Schlaglichtartig seien hier einige Merkmale genannt. Zu diesen gehört, dass Lehrerinnen und Lehrer sich von den performativen Künsten inspirieren lassen, insbesondere von der integrativen Kunstform Theater. Der Unterricht ist oft körperbezogen, d.h. Lehrende und Lernende teilen sich nicht nur sprachlich mit, sondern setzen auch zusätzliche Ausdrucksmittel ein, sind mit ,Kopf, Herz, Hand und Fuß' am kreativen Unterrichtsprozess beteiligt. Eine ausschließliche 
SCHEWE, Manfred - Fremdsprachen performativ lehren und lernen. Para lá da tarefa: implicar os estudantes na aprendizagem de línguas estrangeiras no ensino superior. Porto: FLUP, 2019, pp. 343-361 DOI: https://doi.org/10.21747/9789898969217/paraa19

Fixierung auf Resultate bzw. ,Learning Outcomes‘ wird als problematisch angesehen, vielmehr wird verstärkte Aufmerksamkeit auf die konkreten Lehr- und Lernhandlungen gerichtet, aus denen Unterrichtsinhalte erst entstehen. Im Unterricht kommt es immer wieder zu Rollen- und Perspektivenwechsel, wodurch die Befähigung zur Empathie bzw. auch eine demokratische (interkulturelle/transnationale) Partizipationsfähigkeit gefördert wird. Der Unterricht findet idealerweise in Räumen statt, in denen Unterrichtende und Lernende sich frei bewegen können. Angestrebt wird ein Unterricht in angemessenen Zeitblöcken, so dass tieferes, nachhaltiges Lernen ermöglicht wird. Performatives Lehren und Lernen bedeutet ein Umdenken im Bereich Leistungsbewertung: Es geht weniger um das Sanktionieren von Fehlleistungen im Unterricht, vielmehr werden Fehler als Lern- und Erfahrungschancen begriffen. Nicht zuletzt trägt ein solcher Unterricht zur ,performativen Alphabetisierung' bei, d.h. zur kritischen Auseinandersetzung mit den medialen Aspekten unserer Inszenierungsgesellschaften.

Aus fremdsprachenpädagogischer Perspektive liegt es nahe, den Begriff ,performativ unmittelbar mit Austins (1962) Publikation How to do things with words in Verbindung zu bringen, die den Weg bereitete für Unterrichtskonzepte, in denen der Handlungsaspekt von Sprache betont wurde, etwa im Rahmen einer kommunikativen Didaktik (Schumann, 2010) oder einer handlungsorientierten Didaktik (Bach \& Timm, 2009). Neu in der fremdsprachendidaktischen Fachdiskussion ist aber, angestoßen durch Vertreter*innen der Drama- und Theaterpädagogik, der explizite Bezug auf die performativen Künste und speziell auf die mit diesen assoziierten Formen kreativen Handelns. Wenn also von ,performativem Lehren und Lernen' die Rede ist, so wird damit insbesondere die künstlerische Akzentuierung des Unterrichts hervorgehoben. Wie bereits an anderer Stelle (Even \& Schewe, 2016, pp. 10-23) ausführlicher dargestellt, enthält der Begriff auch die Elemente form und formativ. Im Begriff ist also die Vision von einem Unterricht enthalten, in dem ästhetische Formgebung eine zentrale Rolle spielt und in dem Lernende formative, d.h. für ihre persönliche Entwicklung wichtige Erfahrungen machen können. Das folgende Assoziogramm vermittelt einen Eindruck von zusätzlichen Bedeutungsebenen des Begriffs ,performativ und unterstreicht den hohen Stellenwert von ästhetischer Erfahrung in einem performativ ausgerichteten Fremdsprachenunterricht. 


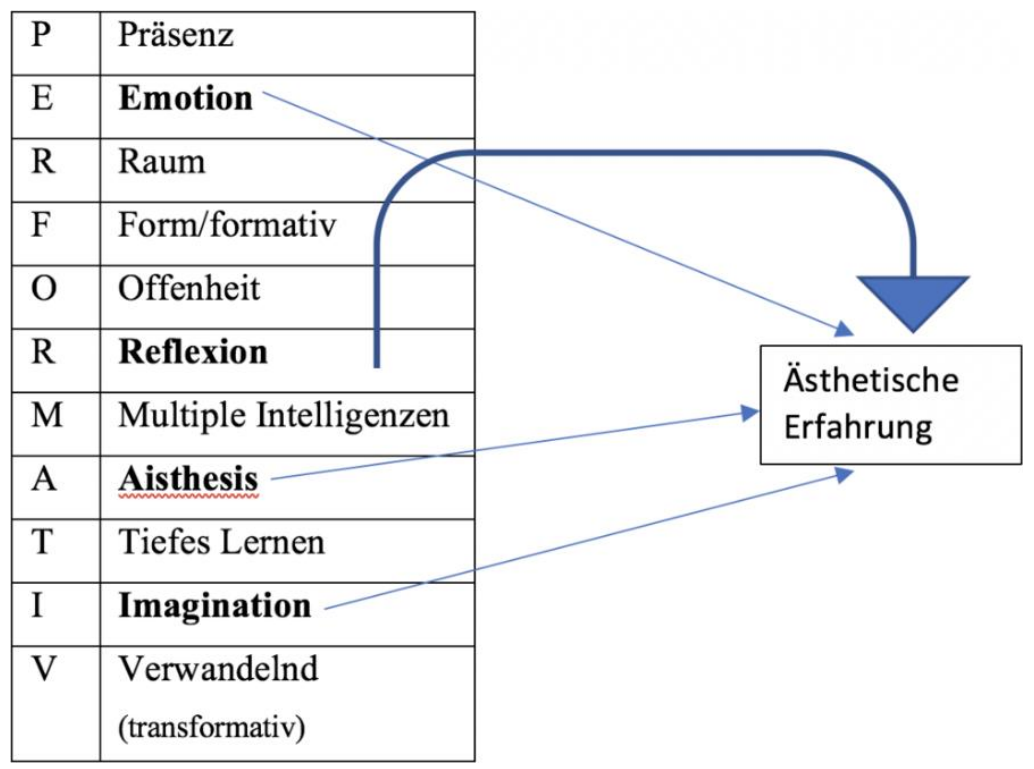

Abbildung 13. Bedeutungsschichten von PERFORMATIV - ein Assoziogramm

Der im Assoziogramm zuerst genannte Begriff ,Präsenż findet im theaterbezogenen Diskurs immer wieder Verwendung. Was im folgenden Zitat über den Akteur gesagt wird, lässt sich auch auf eine Lehrperson, sprich pädagogische Akteurin beziehen: „Wenn von Präsenz des Akteurs die Rede ist, so ist u.a. gemeint, dass er den Raum besetzt und beherrscht, so dass er die Aufmerksamkeit der anderen auf sich zieht“ (Fischer-Lichte, 2012, p. 61). Im performativ gestalteten Unterricht kommen immer wieder Emotionen ins Spiel, z.B. wenn Lernende sich in eine Rolle einfühlen. Ein solcher Unterricht lässt sich am besten in einem Raum durchführen, in dem sich keine sperrigen Möbel befinden und der möglichst viel Bewegungsfreiheit lässt; über den physischen Raum hinaus sind auch die fiktiven Räume zu nennen, in denen Lernende mittels ihrer Vorstellungskraft kreativ handeln. Die Lehrperson schöpft aus dem Formrepertoire der Künste und wird im Laufe der Zeit zum ,Formmeister' bzw. zur ,Formmeisterin“ (Schewe \& Woodhouse, 2018). Offenheit kann bedeuten, dass Unterricht nicht bis ins letzte Detail durchgeplant ist, sondern dass der weitere Gang des Unterrichts immer wieder offen ist und von der Dynamik abhängt, die aus kreativen Unterrichtshandlungen entsteht. Im Unterricht wird immer wieder Gelegenheit zur Reflexion über das Erlebte und neu Gelernte gegeben. Es geht im performativen Unterricht nicht ausschließlich um die Förderung von verballinguistischer Intelligenz, sondern möglichst viele der multiplen Lernenden-Intelligenzen kommen ins Spiel, z.B. die kinästhetische, intra- und interpersonale (zur Theorie multipler Intelligenzen s. Gardner (1993). Der Begriff Aisthesis bezieht sich auf die Sensibilisierung von sinnlicher Wahrnehmung (vgl. dazu insbesondere die oben erwähnte Studie von Huber (2003)). Dadurch, dass Lernende ihre multiplen Intelligenzen 
SCHEWE, Manfred - Fremdsprachen performativ lehren und lernen. Para lá da tarefa: implicar os estudantes na aprendizagem de línguas estrangeiras no ensino superior. Porto: FLUP, 2019, pp. 343-361 DOI: https://doi.org/10.21747/9789898969217/paraa19

einbringen und möglichst viele Sinne aktivieren, erhöht sich die Chance, dass persönlich bedeutsames, tiefes Lernen stattfindet. Und indem Lernende ihre Imagination ins Spiel bringen, können sie über die engen Grenzen ihrer Alltagserfahrung hinausgehen und im geschützten Raum der Fiktion Erfahrungen machen, die verwandelnde Wirkung haben können. Gemeint sind ästhetische Erfahrungen, die - wie im Assoziogramm dargestellt - aus dem besonderen Zusammenspiel von Emotion, Imagination und sinnlicher Wahrnehmung entstehen, wobei das Denken, sprich die Reflexion, stets mit dabei ist. In diesem Zusammenhang sei auf die Perspektive von Fischer-Lichte (2004, p. 341) verwiesen: „So wie die Inszenierung von Theateraufführungen als intendierte performative Hervorbringung ihrer Materialität auf eine Wiederverzauberung der Welt zielt, strebt ästhetische Erfahrung als Schwellenerfahrung nach einer Verwandlung der an der Aufführung Beteiligten. Verwandlung erweist sich so als eine grundlegende Kategorie einer Ästhetik des Performativen.“

\section{4 - Umriss einer performativen Fremdsprachendidaktik}

Performatives Lehren und Lernen ist in einem größeren wissenschaftstheoretischen Kontext zu betrachten. Zum Beispiel setzt sich Bachmann-Medick (2010) aus kulturwissenschaftlicher Perspektive mit der performativen Wende auseinander und Wulf \& Zirfas (2007) beleuchten, in welcher Weise der auf das Performative gerichtete kultur- und sozialwissenschaftliche Diskurs sich auf pädagogische Handlungsfelder beziehen lässt. Die wissenschaftstheoretischen Perspektiven können hier nicht ausführlicher dargestellt werden, im Folgenden soll aber anhand der Abbildung eine erste Vorstellung davon vermittelt werden, was unter einer performativen Fremdsprachendidaktik zu verstehen ist. 
SCHEWE, Manfred - Fremdsprachen performativ lehren und lernen. Para lá da tarefa: implicar os estudantes na aprendizagem de línguas estrangeiras no ensino superior.

Porto: FLUP, 2019, pp. 343-361

DOI: https://doi.org/10.21747/9789898969217/paraa19

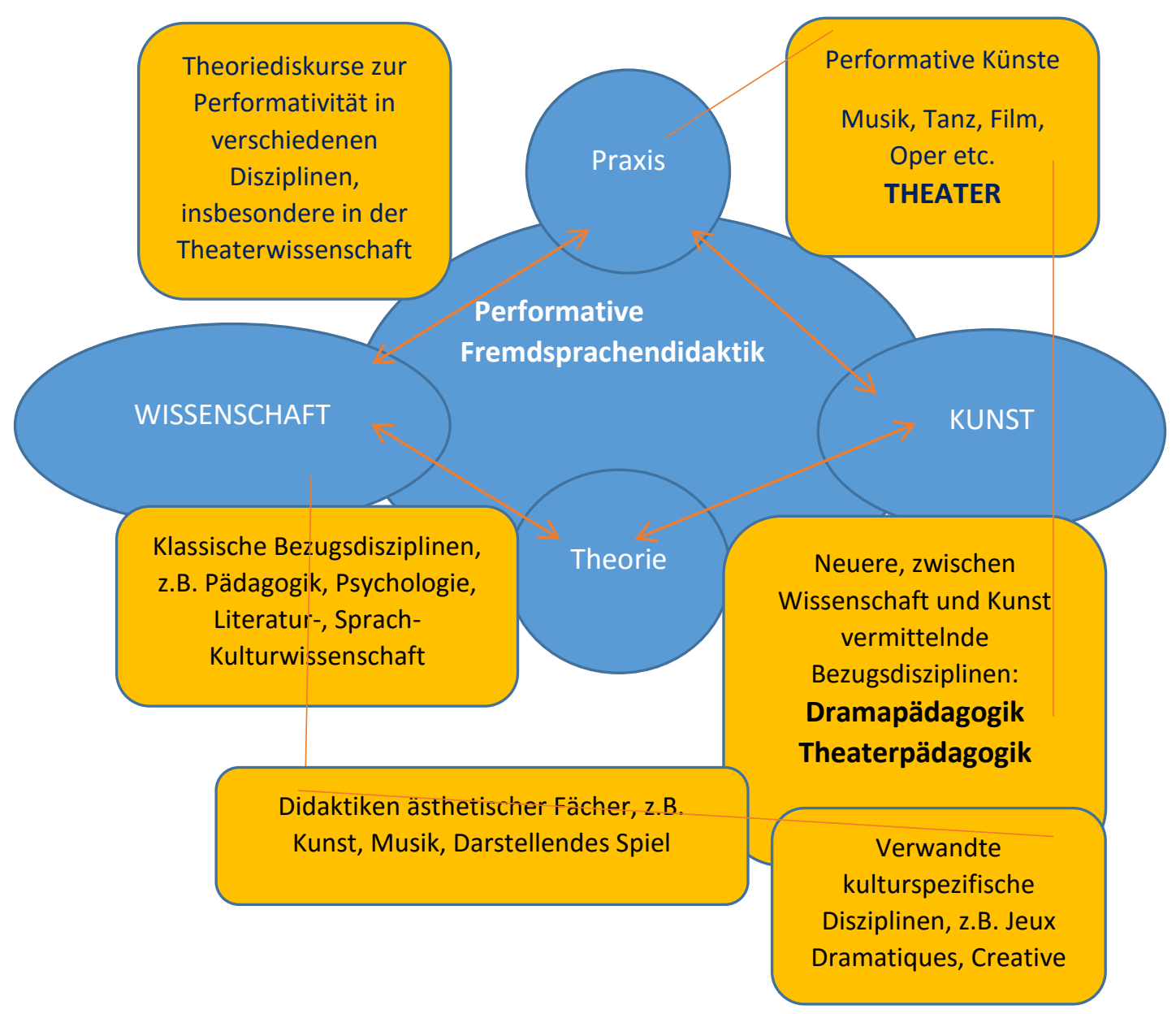

Abbildung 2: Modell einer Performativen Fremdsprachendidaktik - Schewe (2019)

Zentraler Gegenstand der wissenschaftlich orientierten Fremdsprachendidaktik ist die Theorie und Praxis des Lehrens und Lernens. Eine performativ ausgerichtete Fremdsprachendidaktik setzt sich mit dem gleichen Gegenstand auseinander, nähert sich diesem aber aus wissenschaftlicher und künstlerischer Perspektive. Es liegt die Auffassung zugrunde, dass eine qualitative Verbesserung des Lehrens und Lernens von Fremdsprachen erreicht werden kann, wenn sich die Fremdsprachendidaktik mit theater-, sozial- und kulturwissenschaftlichen Diskursen zur Performativität auseinandersetzt und sich stärker zu den performativen Künsten hin öffnet. Diese Auffassung wird inzwischen von vielen Fremdsprachenpädagog*innen geteilt, zum Beispiel vom britischen Dramapädagogen Mike Fleming:

The value of using the performing arts in modern language teaching is clear. It brings more variety and enjoyment to the learning process and thus increases motivation. By making the classroom experience more intense and engaging it 
SCHEWE, Manfred - Fremdsprachen performativ lehren und lernen. Para lá da tarefa: implicar os estudantes na aprendizagem de línguas estrangeiras no ensino superior. Porto: FLUP, 2019, pp. 343-361 DOI: https://doi.org/10.21747/9789898969217/paraa19

heightens the learner's awareness and focus. It brings a more holistic approach in that it engages the body and emotions as well as the mind. (Fleming, 2016, p. 201)

In einer performativen Fremdsprachendidaktik spielen natürlich die traditionellen Bezugsdisziplinen, etwa Pädagogik und Psychologie, und die enger fachbezogenen Disziplinen Linguistik, Literaturwissenschaft und Kulturwissenschaft nach wie vor eine wesentliche Rolle, aber die neueren, zwischen Wissenschaft und Kunst vermittelnden Disziplinen Drama- und Theaterpädagogik (bzw. deren Äquivalent in anderen kulturspezifischen Kontexten) avancieren zu Kerndisziplinen. Inwiefern diese als Wegbereiter einer performativen Fremdsprachendidaktik zu betrachten sind, wurde an anderer Stelle bereits ausführlicher dargestellt (siehe insbesondere die Beiträge im Kapitel I, Grundlagen einer performativen Fremdsprachendidaktik, in Hallet \& Suhrkamp, 2013, pp. 21-86). Was schulbezogene Kontexte angeht, können wertvolle Impulse für eine performative Fremdsprachenlehre auch von den Didaktiken ästhetischer Fächer wie z.B. Bildende Kunst, Musik und Darstellendes Spiel ausgehen.

Zur weiteren Etablierung einer performativen Fremdsprachendidaktik gehört ihre Verankerung an Hochschulen. In diesem Kontext sei auf Fleiners (2016) Bestandsaufnahme zum Auf- und Ausbau einer performativen Lehr-, Lern- und Forschungskultur im Bereich der hochschulischen Fremd- und Zweitsprachenvermittlung verwiesen bzw. auch auf Haack (2018) zu Aspekten performativer Identitätsarbeit und Professionalisierung im Lehramtsstudium.

\section{5 - Zur Praxis performativen Lehrens und Lernens}

Inzwischen wird in allen klassischen Teilbereichen des Fremdsprachenunterrichts (Sprache, Literatur, Kultur), in verschiedensten institutionellen und kulturellen Kontexten und auf verschiedensten Sprachlernniveaus performativ gelehrt und gelernt. Das belegen diverse Veröffentlichungen, zu denen auch Artikel von Autorinnen und Autoren aus über zwanzig Ländern gehören, die seit 2007 in der Fachzeitschrift Scenario Forschungsprojekte vorgestellt und/oder Einblick in ihre pädagogische Praxis gegeben haben.

Aus der inzwischen großen Fülle der Veröffentlichungen können im Folgenden nur einige berücksichtigt werden, um das breite Spektrum von performativer Praxis anzudeuten. Zunächst ist festzustellen, dass zwischen unterschiedlichen Praxis- 
SCHEWE, Manfred - Fremdsprachen performativ lehren und lernen. Para lá da tarefa: implicar os estudantes na aprendizagem de línguas estrangeiras no ensino superior. Porto: FLUP, 2019, pp. 343-361 DOI: https://doi.org/10.21747/9789898969217/paraa19

Formaten zu unterscheiden ist. Performativ lehren und lernen kann bedeuten, dass Projekte durchgeführt werden, die sehr zeitaufwendig und daher oft nur extracurricular möglich sind, kann aber auch bedeuten, dass im Unterricht performative Aktivitäten stattfinden, die innerhalb einer Unterrichtsstunde bzw. Unterrichtseinheit gut realisierbar sind. Die Differenzierung zwischen performativen Großformen und Kleinformen ist bereits an anderer Stelle erfolgt (s. dazu Schewe 2015, p. 27-30), zur Veranschaulichung sollen hier einige kurze Beispiele genügen. Dabei ist zu beachten, dass die Grenzen zwischen Groß- und Kleinformen fließend sind.

\section{1 - Großformen}

\subsection{1 - Inszenierung eines Theaterstücks}

Das klassische Beispiel für eine Großform ist das Proben und Aufführen eines Theaterstücks vor einem öffentlichen Publikum. Üblicherweise wird nach bestimmten Kriterien gemeinsam mit den Schülern bzw. Studierenden ein Stück ausgewählt und im Laufe eines mehrere Wochen/Monate dauernden Probenprozesses eingeübt. Dies verlangt von den Beteiligten hohe Motivation und enormen Einsatz und daher ist eine solch produktorientierte Großform oftmals nur extracurricular zu realisieren. Inwiefern Lernende im Laufe der Probenarbeit und Aufführungen in fachlicher und persönlicher Hinsicht wertvolle Erfahrungen machen, ist bereits oft dokumentiert worden (z.B. Bourke, 1993; Marini-Maio \& Ryan-Scheutz, 2010). Die Lernenden setzen sich intensiv mit dem literarischen Text auseinander, zumal wenn es darum geht, eine Strichfassung bzw. Spielfassung zu erstellen; in einem solchen Fall wird u.a. geprüft wird, wo Längen bzw. ,Spannungsbremsen' oder Breiten auftreten (narrative Seitenstränge, die möglicherweise unnötig irritieren könnten). Wie man sich dies vorzustellen hat, beschreiben die Dramatikerin und Regisseurin Franz und die Hochschullehrerin Hesse (Franz \& Hesse, 2011) am Beispiel ihrer mehrjährigen gemeinsamen Arbeit mit Studierenden der Anglistik. Sie stellen ein mehrphasiges Konzept für die Einstudierung von Theaterstücken vor und beziehen sich dabei u.a. auf das ästhetische Erlebnis des ersten kollektiven lauten Lesens: „[...] die Worte werden Klang, die Leerzeichen werden zu Stille, der Punkt reizt zu körperlicher Reaktion. Wir beginnen, den Text zu fühlen“ (2011, p. 107). Die Aufführung von Stücken in voller Länge ist allerdings eine nicht zu unterschätzende Herausforderung für Schüler bzw. Studierende. Sie agieren schließlich in der Fremdsprache und sind keine professionellen Schauspieler, die aufgrund ihrer großen Erfahrung und Bühnenpräsenz ein Publikum über eine längere Zeitstrecke in Bann halten können. 
SCHEWE, Manfred - Fremdsprachen performativ lehren und lernen. Para lá da tarefa: implicar os estudantes na aprendizagem de línguas estrangeiras no ensino superior. Porto: FLUP, 2019, pp. 343-361 DOI: https://doi.org/10.21747/9789898969217/paraa19

\subsection{2 - Stückerarbeitung}

Eine Alternative zur werkgetreuen Inszenierung eines (kanonischen) dramatischen Textes sind Eigenproduktionen, in denen Texte kreativ miteinander in Beziehung gesetzt und bearbeitet werden. In seinem Artikel „Eigene Texte inszenieren - Englische Theaterproduktionen in der Oberstufe" stellt Poreda (2011) die verschiedenen Phasen der Erarbeitung und Inszenierung einer Tragikomödie moderner Prägung vor, „die insbesondere Themen des jungen Erwachsenenalters behandelt und in Kooperation mit Studenten eines englischen Didaktikseminars auf seine wirkungsästhetische Qualität hin evaluiert wurde“ (Poreda, 2011, p. 132). Er konstatiert dabei u. a. einen Zuwachs an kulturellem und funktionalem literaturwissenschaftlichem Wissen.

Nicht immer sind allerdings dramatische Texte der Impuls und Bezugspunkt für die Erarbeitung eigener Texte. Walter (2011) z.B. stellt ein Erzähltheater-Projekt vor, das mit einer heterogen Gruppe ausländischer Germanistik-Studierenden im Rahmen einer Lehrveranstaltung an der Humboldt-Universität Berlin durchgeführt wurde. Judith Hermanns Prosatext „Sommerhaus, später“ wurde zum Bezugspunkt für das Projekt. Ilg, Kutzelmann, Massler, Peter \& Theinert (2015) haben sich mit der spezifischen Form des (mehrsprachigen) Lesetheaters auseinandergesetzt. Literarische Texte werden in mehrsprachige dialogische Lesetheaterstücke umgewandelt und werden zum Bezugspunkt für das Training des gestaltenden Vorlesens wie auch für die im Klassenverband abschließende Aufführung von Lesetheatern. Crutchfield (2015) geht es um das «gestaltete Lesen», ihm geht es vor allem um eine Erkundung der Erfahrungen, die Studierende machen, wenn sie ihre selbst verfassten literarischen Texte öffentlich vortragen.

\subsection{3 - Theatre-in-Education-Projekte}

Bei einem Theatre-in-Education-Projekt handelt es sich um eine Großform, die meist mit der Aufführung eines von (semi-)professionellen teacher-actors speziell entwickelten Stückes verbunden ist, das möglichst auf Themen ausgerichtet wird, die im Laufe des Schuljahres im Fremdsprachenunterricht noch behandelt werden. Aita (2009) hat am Beispiel Österreichs gezeigt, welch enormen Beitrag Theatre in Education-Gruppen im Laufe der Jahrzehnte zur Entwicklung des schulischen Fremdsprachenunterrichts geleistet haben. 
SCHEWE, Manfred - Fremdsprachen performativ lehren und lernen. Para lá da tarefa: implicar os estudantes na aprendizagem de línguas estrangeiras no ensino superior. Porto: FLUP, 2019, pp. 343-361 DOI: https://doi.org/10.21747/9789898969217/paraa19

\subsection{4 - Improtheater-Projekte}

Basierend auf dem Konzept von Johnstone (1993) bieten Improtheater-Gruppen besonders auch für den Bereich Fremdsprachen Performances und Workshops an. Für die Gruppe ARTIG (www.artiges.org) z.B. steht dabei das Erzählen von Geschichten im Mittelpunkt, wobei in den Performances und Workshops diverse Medien eingesetzt werden (Live-Musik, Projektionen etc.). Haftner \& Riedmüller (2017) beleuchten in einem Beitrag unter dem Titel "The Intercultural Surprise: Teaching Improvisational Theatre in Different Cultural Contexts", wie dieser Ansatz in verschiedenen kulturellen Kontexten aufgenommen wurde.

\subsection{5 - Zielgruppenspezifische theaterpädagogische Workshops}

An sehr vielen deutschen Theatern gibt es inzwischen theaterpädagogische Abteilungen, die für den Brückenbau zwischen Theater und Schule zuständig sind und auf bestimmte Zielgruppen zugeschneiderte theaterpädagogische Workshops anbieten. Jogschies \& Krohn (2009) beschreiben z. B., wie ausländische Germanistik-Studierende an der Universität Hamburg im Rahmen einer Studienreise am Gorki Theater Berlin an einem Workshop teilnahmen, in dem sie durch praktische Theaterarbeit auf ein im Theater gerade aufgeführtes Stück vorbereitet und dabei in deutschlandkundliche Fragestellungen eingeführt wurden.

\subsection{6 - Sprachcamps/ PASCH-Projekte}

$\mathrm{Zu}$ den Großformen gehören weiterhin Sprachcamps, in denen während einer mehrwöchigen Ferienfreizeit Sprachförderung, Theater- und Freizeitaktivitäten ineinandergreifen, wobei die Zwei- bzw. Mehrsprachigkeit der Kinder explizit berücksichtigt wird (vgl. z.B. Stings (2012) Beschreibung des Hamburger TheaterSprachCamp-Modells); oder auch Projekte zwischen Partnerschulen (PASCHProjekte) mit explizitem Fokus auf Theaterarbeit (z. B. Holl, 2011).

\subsection{7 - Dramatische Kulturtransfer-Projekte}

Solchen Projekten liegt die Grundidee zugrunde, dass Fremdsprachenabteilungen als Brückenbauer zwischen verschiedenen Kulturen engagieren, indem dramatische Texte übersetzt, adaptiert und als Vorlage für Inszenierungen vorbereitet werden. Beispiel dafür ist ein an der Universität Cork/Irland realisiertes Projekt (Boyd \& Schewe, 2012), 
SCHEWE, Manfred - Fremdsprachen performativ lehren und lernen. Para lá da tarefa: implicar os estudantes na aprendizagem de línguas estrangeiras no ensino superior. Porto: FLUP, 2019, pp. 343-361 DOI: https://doi.org/10.21747/9789898969217/paraa19

in dem irische Germanistik- sowie Drama und Theatre Studies-Studierende zunächst performative Zugänge zu dem vom Schweizer Dramatiker Thomas Hürlimann (2007) verfassten Stück Das Einsiedler Welttheater erprobten.

\subsection{8 - Poesiewettstreit (Poetry Slam)}

Zunehmend beliebter werden literarische Vortragswettbewerbe, wobei ein selbst verfasster Text einem Publikum lebendig vorgetragen wird. Der/die Vortragende setzt ganz bewusst performative Elemente ein, um das Publikum in Bann zu halten und bewegt sich dabei zwischen Literatur, Theater, Rap und Kabarett. Köhle \& Medusa (2017) beschreiben Workshops und Projekte, in denen Schüler/innen praktisch erproben, „was sich in fünf Minuten auf der Bühne mit Sprache machen lässt“ (ebd., 96). Die Vorbereitung von solch kleinformatigen Performances lässt sich zum Teil auch in den alltäglichen Unterricht integrieren.

\section{2 -Performative Kleinformen}

$\mathrm{Zu}$ den Kleinformen gehören performative Aktivitäten, die im Rahmen einer Unterrichtsstunde bzw. -einheit realisierbar sind, wobei eine inhaltlich anspruchsvolle Unterrichtsgestaltung im Sinne von process drama (Bowell \& Heap, 2013) bedeutet, dass die Lehrperson und Lernenden in wechselnden Funktionen als Akteure, Regisseure, Dramatiker bzw. auch Zuschauer beteiligt sind. Im Laufe des Unterrichts entstehen immer wieder Handlungsprodukte in Form sinnlich erfahrbarer Gestaltungen, bei deren Vorbereitung, Präsentation und Reflexion verbal und nonverbal intensiv gehandelt wird und Lernende in vielfältiger Weise ihr fremdsprachenbezogenes Wissen und Können einbringen bzw. auch systematisch erweitern können. Als Beispiel sei hier die relativ schnell erlernbare Technik Standbild genannt, die im Unterricht vielfältig eingesetzt werden kann. In einem Standbild zeigen die Lernenden (und evtl. auch die Lehrperson) - wie auf einem Foto oder einem angehaltenen Film - eine bestimmte Körperhaltung, Gestik und Mimik, z.B. um darzustellen, was aus ihrer Perspektive ein Schlüsselmoment in der Kurzgeschichte ist, die gerade im Unterricht gelesen wird. Die diversen Einsatzmöglichkeiten von Standbildern sind von Schewe \& Woodhouse (2018) ausführlich beschrieben und anhand von Filmsequenzen veranschaulicht worden. Wie das Standbild und auch andere Techniken zur Erreichung ganz bestimmter Unterrichtsziele eingesetzt werden können, thematisieren zum Beispiel Oelschläger (2017) und Winkelmann (2017). Wer sich in die Unterrichtsgestaltung im Sinne von 
SCHEWE, Manfred - Fremdsprachen performativ lehren und lernen.

Para lá da tarefa: implicar os estudantes na aprendizagem de línguas estrangeiras no ensino superior. Porto: FLUP, 2019, pp. 343-361

DOI: https://doi.org/10.21747/9789898969217/paraa19

process drama weiter einarbeiten möchte, findet bei Piazzoli (2018) viele wertvolle Anregungen. Sie liefert eine wichtige theoretische Fundierung performativen Lehrens und Lernens, veranschaulicht ihr Konzept anhand von diversen Praxisbeispielen und setzt sich dabei speziell mit dem Aspekt 'Teacher Artistry' auseinander.

\section{6 - Ausblick}

Die Disziplinen Drama- und Theaterpädagogik haben bewirkt, dass sich die Fremdsprachendidaktik zu den performativen Künsten hin weiter öffnet. Damit ist ein spannendes, neues Praxis- und Forschungsfeld entstanden. Inzwischen liegen kritischempirische Forschungsarbeiten vor, z.B. Müllers (2017) Studie zur sprachlichen Kognitivierung im dramapädagogischen DaF-Unterricht, und aus neurowissenschaftlicher Perspektive wird zunehmend auch die besondere Effizienz eines performativ gestalteten Unterrichts untermauert (Sambanis \& Walter, 2019).

Es bleibt weiter zu erkunden, in welcher Hinsicht der Körper ein wichtiges Kommunikations- und Erkenntnismedium ist, in welchem Sinne Fremdsprachenunterricht eine eigene Ästhetik haben kann und wie Lehrer*innen in der Aus- und Fortbildung künstlerische Handlungskompetenzen entwickeln können.

\section{Literaturverzeichnis}

Aita, S. (2009). The Theatre in Language Learning (TiLL) Model. Exploring Theatre as Pedagogy in the L2 Environment. Scenario III, 1, 64-80.

Anderl, S. (2019). Welche Sprachen spricht die Kunst? Frankfurter Allgemeine Zeitung (6. März 2019). https://www.faz.net/aktuell/feuilleton/debatten/was-sich-kunstund-wissenschaft-zu-sagen-haben-16073702.html.

Austin, J. L. (1962). How to do things with words. Oxford: Clarendon.

Bach, G. \& Timm , J.-P. (2009). Englischunterricht: Grundlagen und Methoden einer handlungsorientierten Unterrichtspraxis. Tübingen: Francke.

Bachmann-Medick, D. (2010). Cultural Turns. Neuorientierungen in den Kulturwissenschaften. Reinbek: Rowohlt.

Bernstein, N. \& Lerchner, C. (Hrsg.) (2014). Ästhetisches Lernen im DaF-/DaZUnterricht. Literatur - Theater - Bildende Kunst - Musik - Film. Göttingen: Universitätsverlag. 
SCHEWE, Manfred - Fremdsprachen performativ lehren und lernen. Para lá da tarefa: implicar os estudantes na aprendizagem de línguas estrangeiras no ensino superior. Porto: FLUP, 2019, pp. 343-361 DOI: https://doi.org/10.21747/9789898969217/paraa19

Bourke, E. (1993). Work at the Coalface: An Empirical Approach to Foreign Language Theatre for Students. In: Manfred Schewe \& Peter Shaw (Hrsg.): Towards Drama as a Method in the Foreign Language Classroom. Frankfurt a.M.: Peter Lang, 227-248.

Bowell, P. \& Heap, B. S. (2013). Planning Process Drama. Enriching Teaching and Learning. Abingdon: Routledge.

Boyd, S. \& Schewe, M. (2012). Welttheater: übersetzen, adaptieren, inszenieren World Theatre: translation, adaptation, production. Berlin: Schibri.

Crutchfield, J. (2015). Creative Writing and Performance in EFL Teacher Training: A Preliminary Case Study. Scenario IX, 1, 3-34.

Crutchfield, J. \& Schewe, M.(Hrsg.) (2017). Going Performative in Intercultural Education. International Contexts, Theoretical Perspectives and Models of Practice. Bristol: Multilingual Matters.

Even, S. (2002). Drama Grammatik. Dramapädagogische Ansätze für den Grammatikunterricht Deutsch als Fremdsprache. München: iudicium.

Even, S. \& Schewe, M. (2016). Performatives Lehren, Lernen und Forschen Performative Teaching, Learning, Research. Berlin: Schibri.

Fischer-Lichte, E. (2004). Ästhetik des Performativen. Frankfurt a.M.: Suhrkamp.

Fischer-Lichte, E. (2012). Performativität. Eine Einführung. Bielefeld: Transcript.

Fleiner, M. (2016). Performancekünste im Hochschulstudium: Transversale Sprach-, Literatur- und Kulturerfahrungen in der fremdsprachlichen Lehrerbildung. Berlin: Schibri.

Fleming, M. (2018). Performativity, Learning and Aesthetic Education. In: Olivier Mentz, O \& Fleiner, M. The Arts in Language Teaching. International Perspectives: Performative - Aesthetic - Transversal. Zürich: LIT Verlag, 6-20.

Franz, S. \& Hesse, M. (2011). Vom Lesen eines Prosatextes bis zur dramatischen Aufführung: ein Gang durch verschiedene Rezeptionsebenen. In: Almut Küppers, Torben Schmidt \& Maik Walter (2011): Inszenierungen im Fremdsprachenunterricht. Grundlagen, Formen, Perspektiven. Braunschweig: Schroedel/Diesterweg/Klinkhardt, 104-116.

Gardner, H. (1993). Multiple Intelligences. The Theory in Practice. A Reader. New York: Basic Books.

Haack, A. (2018). Dramapädagogik, Selbstkompetenz und Professionalisierung. Performative Identitätsarbeit im Lehramtsstudium Englisch. Stuttgart: Metzler.

Haftner, M. \& Riedmüller, A. (2017). The Intercultural Surprise: Teaching Improvisational Theatre in Different Cultural Contexts. In: Crutchfield, J. \& Schewe, M. 
SCHEWE, Manfred - Fremdsprachen performativ lehren und lernen. Para lá da tarefa: implicar os estudantes na aprendizagem de línguas estrangeiras no ensino superior. Porto: FLUP, 2019, pp. 343-361 DOI: https://doi.org/10.21747/9789898969217/paraa19

(Hrsg.): Going Performative in Intercultural Education. International Contexts, Theoretical Perspectives and Models of Practice. Bristol: Multilingual Matters, 3-20.

Hallet, W. \& Surkamp, C. (Hrsg.) (2015). Dramendidaktik und Dramapädagogik im Fremdsprachenunterricht. Handbuch 5. Handbücher zur Literatur- und Kulturdidaktik. Trier: Wissenschaftlicher Verlag.

Hensel, A. (in Vorbereitung). Fremdsprachenunterricht als Ereignis - Zur Fundierung einer performativ-ästhetischen Praxis. Dissertation, Freie Universität Berlin.

Holl, E. (2011). SPRACH-FLUSS: Theaterworkshops mit Jugendlichen aus 16 afrikanischen Ländern - Theaterpädagogik zwischen kultureller Bildung und Fremdsprachendidaktik. Scenario V, 2, 13-31.

Huber, R. (2003). Im Haus der Sprache wohnen. Wahrnehmung und Theater im Fremdsprachenunterricht. Tübingen: Niemeyer.

Hürlimann, T. (2007). Das Einsiedler Welttheater. Zürich: Ammann.

Ilg, A., Kutzelmann, S., Massler, U., Peter, K. \& Theinert, K. (2015). Dramapädagogische Elemente im Leseförderprojekt „Mehrsprachiges Lesetheater“ (MELT). Scenario IX, 2, 42-62.

Johnstone, K. (1989). Impro. Improvisation and the Theatre. London: Methuen.

Jogschies, B. \& Krohn, D. (2009). Heaven - Theaterpädagogisch vor- und nachbereitet. Scenario III, 1, 3-16.

Jogschies, B., Schewe, M. \& Stöver-Blahak, A. (2018). Empfehlungen zur Förderung einer performativen Lehr-, Lern- und Forschungskultur an Hochschulen. Scenario XII, 2, 47-51.

Köhle, M. \& Medusa, M. (2017). Gemma Slam! Poetry Slam in der Schule. Informationen zur Deutschdidaktik (IDE) 41, 3, 95-101.

Marini-Maio, N. \& Ryan-Scheutz, C. (Hrsg.) (2010). Set the Stage! Teaching Italian through Theater. Theories, Methods and Practices. New Haven and London: Yale University Press.

Mentz, O. \& Fleiner, M. (Hrsg.) (2018). The Arts in Language Teaching. International Perspectives: Performative - Aesthetic - Transversal. Zürich: LIT Verlag.

Moraitis, A., Mavruk, G., Schäfer A. \& Schmidt, E. (Hrsg.) (2018). Sprachförderung durch kulturelles und ästhetisches Lernen. Sprachbildende Konzepte für die Lehrausbildung. Münster: Waxmann.

Müller, T. (2017). Sprachliche Kognitivierung im dramapädagogischen Deutsch-alsFremdsprache-Unterricht. Eine Bestandsaufnahme und empirische Expertenbefragung. München: iudicium. 
SCHEWE, Manfred - Fremdsprachen performativ lehren und lernen. Para lá da tarefa: implicar os estudantes na aprendizagem de línguas estrangeiras no ensino superior. Porto: FLUP, 2019, pp. 343-361 DOI: https://doi.org/10.21747/9789898969217/paraa19

Oelschläger, B. (2017). Bühne frei für Deutsch! Das Theaterhandbuch für Deutsch als Fremdsprache. Weinheim: Deutscher Theaterverlag.

Piazzoli, E. (2018). Embodying Language in Action. The Artistry of Process Drama in Second Language Education. Cham/CH: Palgrave Macmillan.

Poreda, W. (2011). Eigene Texte inszenieren - Englische Theaterproduktionen in der Oberstufe. Almut Küppers, Torben Schmidt \& Maik Walter (2011): Inszenierungen im Fremdsprachenunterricht. Grundlagen, Formen, Perspektiven. Braunschweig: Schroedel/Diesterweg/Klinkhardt, 131-140.

Sambanis, M. \& Walter, M. (2019). In Motion - Theaterimpulse zum Sprachenlernen. Von neuesten Befunden der Neurowissenschaft zu konkreten Unterrichtsimpulsen. Berlin: Cornelsen.

Schewe, M. (1993). Fremdsprache inszenieren. Zur Fundierung einer dramapädagogischen Lehr- und Lernpraxis. Oldenburg: Didaktisches Zentrum.

Schewe, M. (1997). DaF-Lehrerlnnen-Ausbildung: nicht nur als Wissenschaft, sondern ebenso als Kunst. A. Wolff, G. Tütken \& H. Liedtke (Hrsg.). Gedächtnis und Sprachlernen; Prozeßorientiertes Fremdsprachenlernen; Deutschlehrerausbildung in West- und Osteuropa; Eine deutsche Literatur: AutorInnen nichtdeutscher Muttersprache. Regensburg: Fachverband Deutsch als Fremdsprache, 245-254.

Schewe, M. (2014). Für das Ästhetische einen Ort schaffen. DaF als Bauhaus - ein Vorentwurf. Bernstein, N. \& Lerchner, C. (Hrsg.): Ästhetisches Lernen im DaF-/DaZUnterricht. Literatur - Theater - Bildende Kunst - Musik - Film. Göttingen: Universitätsverlag Göttingen.

Schewe, M. (2015). Fokus Fachgeschichte: Die Dramapädagogik als Wegbereiterin einer performativen Fremdsprachendidaktik. W. Hallet \& C. Surkamp (Hrsg.). Dramendidaktik und Dramapädagogik im Fremdsprachenunterricht. Handbuch 5. Handbücher zur Literatur- und Kulturdidaktik. Trier: Wissenschaftlicher Verlag, 21-36.

Schewe, M. (2018). Der Traum vom leeren Raum: Perspektiven einer performativen Lehr-, Lern- und Forschungskultur. Scenario XII, 2, 63-67.

Schewe, M. \& Woodhouse, F. (2018). Performative Foreign Language Didactics in Progress: About Still Images and the Teacher as 'Formmeister' (Form Master). Scenario XII, 2, 53-69.

Schmenk, B. (2017). Myths of Origin and the Communicative Turn. Critical Multilingualism Studies 5(1), 7-36.

Schumann, A. (2010). Kommunikativer Fremdsprachenunterricht. C. Surkamp (Hrsg.): Lexikon Fremdsprachendidaktik. Stuttgart: Metzler, 137-140. 
SCHEWE, Manfred - Fremdsprachen performativ lehren und lernen.

Para lá da tarefa: implicar os estudantes na aprendizagem de línguas estrangeiras no ensino superior.

Porto: FLUP, 2019, pp. 343-361

DOI: https://doi.org/10.21747/9789898969217/paraa19

Sting, W. (2012). Performance und Theater als anderes Sprechen. Scenario VI, 1, 54-63.

Tselikas, E. I. (1999). Dramapädagogik im Sprachunterricht. Zürich: Orell Füssli.

Walter, M. (2011). Prosa in Szene setzen - Generation X trifft Generation Harry Potter.IN: A. Küppers, T. Schmidt \& M. Walter (2011): Inszenierungen im Fremdsprachenunterricht. Grundlagen, Formen, Perspektiven. Braunschweig: Schroedel/Diesterweg/Klinkhardt, 131-140.

Winkelmann, U. (2015). Vergnüglich Lernen! Ein Praxisbuch über die Lehr- und Lernmethode Dramapädagogik. Mit 10 Unterrichtsbeispielen für die Sekundarstufen I und II. Berlin: Verlag Vergnügliches Lernen.

Wulf, C. \& Zirfas, J. (Hrsg.). Pädagogik des Performativen. Theorien, Methoden, Perspektiven. Weinheim: Beltz.

Online-Quellen

28. Kongress der Deutschen Gesellschaft für Fremdsprachenforschung (DGFF) in Würzburg (25.-28.9.2019) - https://www.dgff.de/veranstaltungen/dgff-kongress/ [5.4.2019].

UNESCO First World Conference on Arts Education - Roadmap for Arts Education, 2006 http://www.unesco.org/new/fileadmin/MULTIMEDIA/HQ/CLT/CLT/pdf/Arts_Edu_ RoadMap_en.pdf [10.01.2019].

UNESCO Second World Conference on Arts Education, 2010 http://www.unesco.org/new/en/culture/themes/creativity/arts-education/worldconferences/2010-seoul/ [10.01.2019].

UN Sustainable Development Goals, https://www.un.org/sustainabledevelopment/sustainable-development-goals/ [5.3.2019]. 